\title{
Die Ranunculaceae der Flora von Zentraleuropa: Isopyrum ${ }^{1}$
}

\author{
ECKEHART J. JÄGER, Martin-Luther-Universität 06108 Halle, Neuwerk 21, \\ e-mail: jaegerbot@gmail.com
}

\section{Isopyrum $^{2}$}

LINNAEUS 1753, Sp. P1. 557. nomen conservandum- Syn.: Olfa ADANSON 1763, Fam. Pl. 2: 458 (nom. superfl.). - Thalictrella A. RICHARD in BORY 1826, Dict. Class. Hist. Nat. 9: 34. - M u s c h e 1 b 1 ü m chen, Tolld ocke. Franz.: isopyre; ital.: isopiro; slowen.: polžarka; ungar.: galambvirág; slowak.: veterník; tschech.: zapalice; poln.: zdrojówka; russ.: равноплодник [ravnoplodnik]. Abb.: KADEREIT \& MELZHEIMER 2021, Tafel 11 Fig. 4 (http://www.flora-deutschlands.de/ranunculaceae.html; Fig. 4c falsch, wohl mit dem Samen von Delphinium verwechselt).

Typus-Art: Isopyrum thalictroides L., typus conserv. (s. Taxon 47 [4]: 871, Prop. 1225 [1998])

Literatur. ClaRKe, G. C. S., PUNT, W \& P. P. HOEN 1991: Ranunculaceae. Northwest European Pollen Flora 51. Rev. Paleobot. Palynol. 69: 117-271. - DRUMMOND, J. R. \& J. HUTCHINSON 1920: A revision of Isopyrum (Ranunculaceae) and its near allies. Kew Bull. 5: 145-169. - FRENCEL, J.M. 1966: Alkaloide von Isopyrum thalictroides. I. Isolierung und Identifizierung von Magnoflorin aus Wurzeln und Rhizomen. Planta Med. 14: 204-208. - GREGORY, W. C. 1941: Phylogenetic and cytological studies in the Ranunculaceae. Juss. Trans. Am. Phil. Soc. N. S. 31: 443-521. - HeNRY, M. et al. 1999: An ecological comparison between ancient and other forest plant species of Europe, and the implications for forest conservation Biol. Cons. 41: 9-22. - HIEPKO, P. \& M. TAMURA 1996: (1225) Proposal to conserve the name Isopyrum L. (Ranunculaceae) with a conserved type. Taxon 45: 327-328 und Taxon 47: 871 (1998). - JALAS, J. \& J. SUOMINEN 1989: Atlas Florae Europaeae, Vol. 8: S. 39. Helsinki: The Committee for mapping the Flora of Europe and Societas Biologica Fennica Vanamo. - KADEREIT, J. W. \& V. MELZHEIMER 2021: Die Ranunculaceae der Flora von Zentraleuropa: Familie Ranunculaceae. - Publiziert unter http://www.flora-deutschlands.de/ranunculaceae.html. - KÄSTNER, A., \& M. A. FISCHER 2006: Porträts ausgewählter seltener österreichischer Gefäßpflanzenarten II (5-15). Neilreichia 4:75-109. - KovAndA, M. 1963: Isopyrum thalictroides L. in Böhmen. (tschech. mit dtsch. Zusfg.)

\footnotetext{
1 Zitierempfehlung: JÄGER, E. J. 2021: Die Ranunculaceae der Flora von Zentraleuropa: Isopyrum . - Publiziert unter http://www.flora-deutschlands.de/ranunculaceae.html

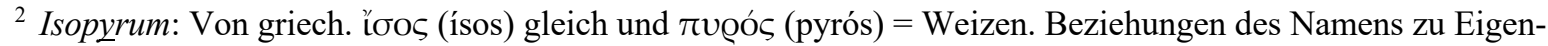
schaften der Pflanze sind unbekannt. Der Name Isopyrum ist bei DiOsKURIDES, Mat. med. 4, 120 und PLINIUS, Hist. Nat. 27, 94, der Name für eine Pflanze mit bohnenähnlichen Früchten. LiNNAEUs (1753) übertrug den Namen auf unsere Gattung.
} 
Preslia 35: 217-223. - KROTOSKA, I. \& H. PIOTROVSKA 1959: Isopyrum thalictroides in the lowland of Wielkopolska and Kujawy (poln. mit engl. Zusfg.) Fragm. Flor. Geobot. 5: 357-363. - LANGLET, O. 1932: Über die Chromosomenverhältnisse und Systematik der Ranunculaceae. Sv. Bot. Tidskr. 26: 381400. - MARZELL, H. 1972: Wörterbuch der deutschen Pflanzennamen, Band 2. Leipzig: Hirzel. MASU, J. 1954: On the pollen-grains of Isopyrum (Ranunculaceae) (jap. mit engl. Zusfg.) Jour. Jap. Bot. 29: 331-333. - MELZER, H. 1984: Neues zur Flora der Steiermark, XXVI. Isopyrum thalictroides L. var. pubescens WIERZB. Mitt. Naturwiss. Ver. Steiermark 114: 251-252. - MLADÝ, F. \& J. KOLBEK 1976: Chorologisch-phytozönologische Probleme der Verbreitungsgeschichte von Isopyrum thalictroides in der Umgebung von Křivoklát. Preslia (Praha) 48: 143-155 (tschech. mit dt. Zusammenfassung). NARDI, E. 1993: Problems of typification of the genus Isopyrum L. (Ranunculaceae). Webbia 47: 227-231. - OPREA, I. V. 1965: An evolution case history of the perianth of Isopyrum thalictroides L. f. pubescens (WierzB.) JAV. (rum. mit engl. Zusfg.) Comun. Bot. (Bucuresti) 3: 137-141. - POMPE, S. \& (10) al.: 2011: Modellierung der Auswirkung des Klimawandels auf die Flora und Vegetation in Deutschland. Bundesamt für Naturschutz, Bonn - Bad Godesberg 2011. - SCHÖNFELDER, P. 1971: Südwestliche Einstrahlungen in der Flora und Vegetation Nordbayerns. Ber. Bayer. Bot. Ges. 42: 17-100. SKALINSKÁ, M. 1958: Seed development after crosses of Aquilegia with Isopyrum. Stud. Pl. Physiology, Praha 1958: 213-221; 1958: Some attempts to obtain hybrids between Aquilegia and Isopyrum. Proc. X. Int. Congr. Genet. 2: 263. - TAKEDA, H. 1968: On some morphological characteristics of the genus Isopyrum. Jour. Jap. Bot. 43: 368-371. - TAMURA, M. 1993: Ranunculaceae. In: KUBITZKI, K. (Ed.) Families and genera of vascular plants. Vol. 2: 563-583. Berlin: Springer. - TAMURA, M. 1995: (Ranunculaceae) General and systematic part. In: HIEPKO, P. (Ed.): Die natürlichen Pflanzenfamilien. 17 a IV, Ordnung Ranunculales, Familie Ranunculaceae. 2. Aufl. Berlin: Duncker \& Humblot. - TAMURA, M. \& L. A. LAUENER 1968: A revision of Isopyrum, Dichocarpum and their allies. Notes Roy. Bot. Gard. Edinburgh 28: 267-273. - TAMURA, M \& K. KOSUGE 1989: Classification of the Isopyroideae (Ranunculaceae). Acta Phytotax. Geobot. 40: 31-35. - Troll, W. \& F. WEBERLING 1989: Infloreszenzuntersuchungen an monetelen Familien. Stuttgart, New York: Fischer. (S. 138-140). - WANG, W. \& Z. D. CHEN 2007: Generic level phylogeny of Thalictroideae (Ranunculaceae) - implications for the taxonomic status of Paropyrum and petal evolution. Taxon 56: 811-821. - WANG, W., LU, A,-M., REN, Y., ENDRESS, M.E. \& Z.-D. CHEN 2009: Phylogeny and classification of Ranunculales: Evidence from four molecular loci and morphological data. Perspect. Plant Ecol. 11: 81-110. - WINKLER, A. 1884: Die Keimpflanze des Isopyrum thalictroides L. Flora 67: 195-198.

Niedrige, frühjahrs- bis frühsommergrüne Stauden mit unterirdisch kriechendem Ausläufer-Rhizom, Geophyten. Blätter mehrfach dreiteilig oder fiederig. Blütenstand eine foliose oder bracteose Traube, die einzelnen Blüten meist in den Achseln von laubblattähnlichen Tragblättern. Äußere Blütenhüllblätter (= Kelchblätter) kronblattartig, 5-6, weiß. Innere Blütenhüllblätter (= nektarfertile Kronblätter) 5-6, viel kürzer als die Kelchblätter, mit ihnen abwechselnd, kurz genagelt oder sitzend, flach oder tütenförmig, Staubblätter zahlreich, Fruchtblätter (1-) 2-8 (-10) frei, sitzend.

\section{Abgrenzung und systematische Stellung der Gattung Isopyrum.}

Schon die Tatsache, dass Isopyrum thalictroides mindestens 10 Synonyme hat, verweist auf die bewegte Geschichte der Taxonomie der Gattung. In der ersten Auflage des HEGI wurde der Umfang mit etwa 30 Arten angegeben, in der 2. Auflage nach TAMURA \& LAUENER (1967) unter Einschluss von Paropyrum anemonoides nur mit 4 (s. u.). 
Die Unterfamilie Thalictroideae RAF., die durch Chromosomen vom T(halictrum)-Typ und $\mathrm{x}=7$ charakerisiert ist, umfasst nach WANG \& CHEN 2007 und WANG et al. (2009) 3 Abstammungsgemeinschaften $(\rightarrow$ Abb. 1):
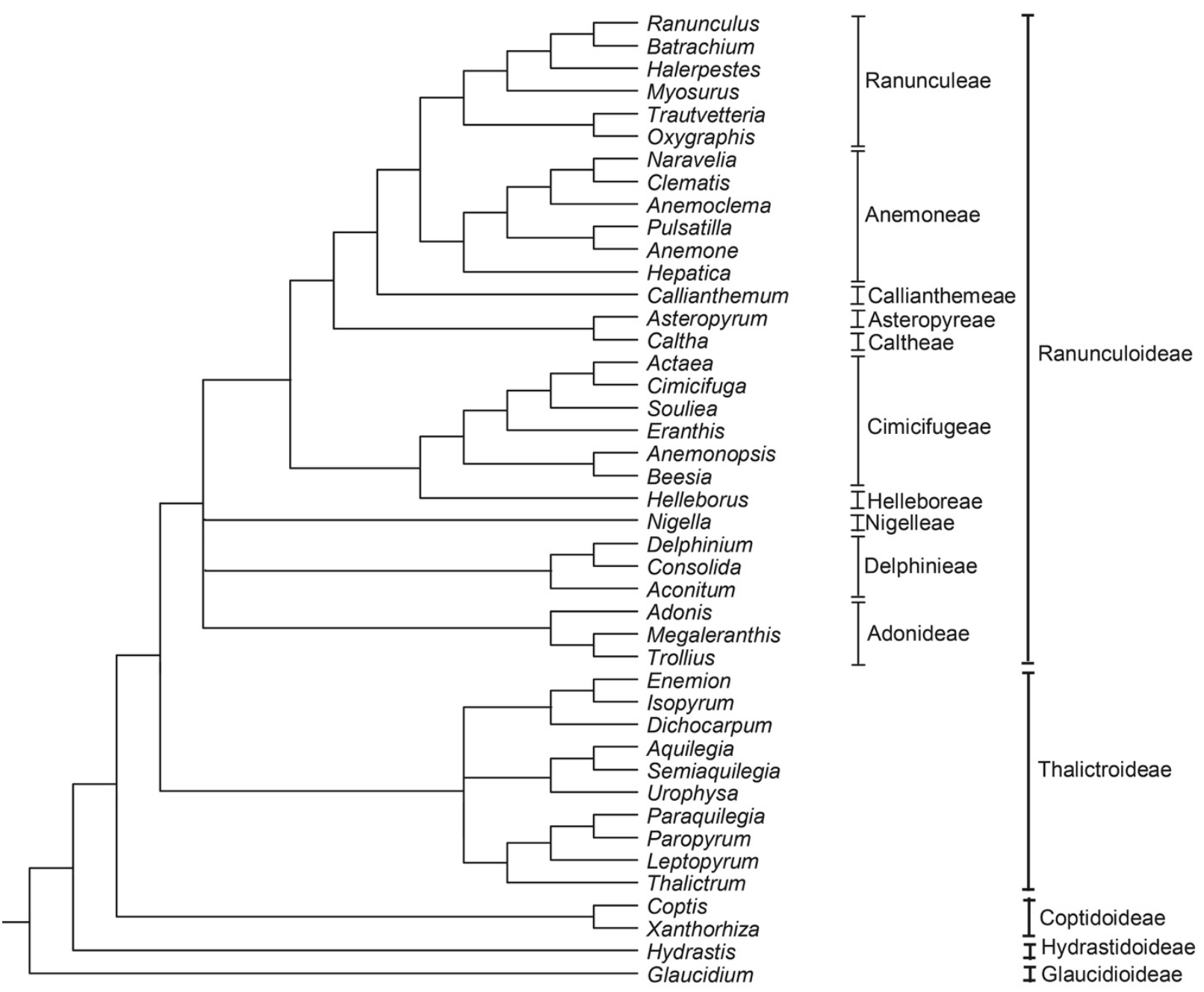

Abb. 1 Verwandtschaftliche Stellung von Isopyrum innerhalb der Thalictroideae (Nach WANG \& al. 2009, Ausschnitt, vgl. auch WANG \& CHEN 2007).

Den ersten Ast des molekularen Stammbaumes bilden Paraquilegia J. R. DRUMM. \& HUTCH. (8 Arten, in Schluchten, auf Felsbändern der hochmontanen und alpinen Stufe von Iran, Pakistan, Mittelasien (Tienschan, Alatau, Pamir-Alai Kasachstan, Mongolei bis russ. Fernost und Japan, m-temp/mo-alp k1-2 M-OAS); Paropyrum O.E. UlbR. (nur P. anemonoides (KAR. \& KIR.) O.E. UlbR.; Syn. Isopyrum anemonoides KAR. et KIR.) (zarte Schattenpflanze in Felsspalten, im Schutz von Felsen, an Bergbächen und Gletschern im Hochgebirge bis 4000(-4500) m, des meridional-submeridionalen Mittel-, Zentralund Ostasiens; von Kasachstan, Turkmenien, Afghanistan, Pakistan, dem Tienschan, dem Pamir und dem westlichen Himalaya über die Mongolei [Altai], Qinghai bis ins nördliche Zentralchina, $\mathrm{m}-\mathrm{sm} / \mathrm{mo}$ alp k1-2 M-OAS); Leptopyrum RCHB. (monotypische Gattung, nur L. fumarioides (L.) RCHB. (Syn.: Isopyrum fumarioides L., Neoleptopyrum fumarioides (L.) HUTCH.), einjährig, Fruchtblätter 10-20, frei, sm-b k1-2 O-EUR-AS) von Osteuropa über fast die ganze Mongolei bis China und Nordkorea, in Bergsteppen) und Thalictrum TOURN. ex L. (etwa 150 Arten, tropisch/montan bis arktisch circumpolar). 
Den zweiten Ast bilden Urophysa O.E. UlBR. (2 Arten in Ost- und Mittelchina, m-sm oz 1-3 OAS); Semiaquilegia MAKINO (nur S. adoxoides (DC.) MAKINO: Japan, Korea, Süd- und Mittelchina; 1001100 m) und Aquilegia L. (s. dort).

Den dritten Ast bilden Dichocarpum W. T. WANG \& HSIAO (18 Arten, japono-himalaisch, früher alle zu Isopyrum), Enemion RAF. (6 Arten, alle - nach WANG \& CHEN (2007) wohl sekundär - ohne Kronblätter, sm-temp oz2-3WAM+OAM, früher ebenfalls zu Isopyrum) und Isopyrum L. als Schwestergattung dieser beiden Gattungen (WANG \& CHEN 2007)

Danach ist Paropyrum also nicht näher mit Isopyrum verwandt, und Isopyrum enthält dann nur 3 Arten. Die Verwandtschaft von Paropyrum zu Paraquilegia und Leptopyrum wird durch die verbreiterten Filamente unterstützt. Bei Isopyrum sind die Filamente fädig (WANG \& CHEN 2007).

Ungeachtet dieser Verwandtschaftsverhältnisse wird Paropyrum vielfach in Isopyrum eingeschlossen (PLANTS OF THE WORLD ONLINE 2021, TAMURA \& LAUENER 1968, TAMURA 1993, 1995, EURO+MED 2006-, dort als Untergattung von Isopyrum).

Die Gattung Isopyrum enthält also 3 Arten, zwei in Ost- und Zentralasien

I. ludlowii TAMURA et LAUENER (West-Himalaya, Kaschmir). (PACHOMOVA 1974 trennt diese Art als neue, monotypische Gattung ab: Alexeya vvedenskyi PACHOM.; nach PlANTS OF THE WORLD ONLINE 2021 ist die letztere aber ein Synonym von Paraquilegia anemonoides (WILLD.) ULBR. und nicht von Isopyrum ludlowii);

I. manshuricum (KOM.) KOM. ex WANG \& HSIAO in (Syn.: Semiaquilegia manshurica KOM.): NOChina (Heilongijang, Jilin, Liaoning) Russ. Fernost (Primorje, Ussuri-Gebiet) und Korea,

und I. thalictroides L. in Europa (s. u.)

EURO+MED (2006-) stellen die Gattung zur Tribus Isopyreae SCHRÖD., die außerdem Aquilegia und Semiaquilegia, aber nicht Thalictrum umfasst.

Zwei weitere asiatische Arten, die früher zu Isopyrum gestellt wurden, sind Isopyrum grandiflorum FISCH. ex DC. (Syn. von Paraquilegia anemonoides (WILLD.) O.E. ULBR., nicht zu verwechseln mit Isopyrum anemonoides KAR. \& KIR. = Paropyrum anemonoides (KAR. \& KIR.) O.E. UlBR.= Paraquilegia anemonoides (KAR. \& KIR.) SCHIPCZ. 1924 non (WILlD.) O.E. UlBR. 1922) und Isopyrum microphyllum ROYLE (Syn. von Paraquilegia. microphylla (ROYLE) J. R. DRUMM. \& HUTCH.).

Wegen der Probleme der Typisierung von Isopyrum L. wurde von NARDI (1993) vorgeschlagen, alle 4 Arten (inkl. I. anemonoides) in die Gattung Thalictrella A. RICHARD zu stellen, der Vorschlag wurde nicht akzeptiert.

Blütenstände. Im Scheitelbereich des Blütentriebes findet sich über den fertilen (achselständige Blüten tragenden) noch ein steriles Hochblatt vor, was anzeigt, dass der Verrumpfung nicht nur die Endblüte zum Opfer gefallen ist, sondern dass das Ende des Blütenstandes in größerem Umfang rudimentiert ist (,Rumfsynfloreszenz“). In der verwandten Gattung Enemion finden sich noch Trauben-ähnliche Blütenstände mit Endblüte (Botryoide). An der Basis des Blütentriebes von I. thalictroides stehen ein oder zwei Grundblätter, die wohl schon zum nächstjährigen Innovationsspross gehören. 
Pollenmorphologie. Pollenkörner vom Caltha palustris-Typ = Actaea-Typ (CLARKE et al. 1991) (s. bei Caltha). Trizonocolpat, manchmal pantocolpat (4 oder mehr Colpi). Colpi lang, mäßig breit. Exine dünn. Sexine etwa 2x so dick wie die Nexine. Deutlich echinat bis mikroechinat, Tectum zwischen den Mikroechini perforiert.

Cytologie. I.thalictroides ist diploid mit $2 \mathrm{n}=14$ Chromosomen. Die Chromosomen sind klein und einfach gekrümmt. Sie entsprechen dem Thalictrum-Typ (GREGORY 1941, LANGLET 1932).

Inhaltsstoffe. Besonders in den unterirdischen Organen von I. thalictroides wurden Benzylisochinolinalkaloide (u.a. Magnoflorin) und Bisbenzylisochinolinalkaloide (Isopyruthaline und Isopythaline) nachgewiesen. Ranunculin fehlt, dafür sind aber cyanogene Verbindungen (u. a. Triglochinin) wie bei Aquilegia, Thalictrum und Leptopyrum gefunden worden, sowie ungesättigte Fettsäuren (z. B. 18:3 $\Delta 5$ t, $9 \mathrm{c}, 12 \mathrm{c})$. I. thalictroides ist giftig.

Krankheiten und Schädlinge. I. thalictroides wird von dem falschen Mehltau Plasmopara isopyrithalictroidis (WARTEW.) SAVUL. auf der Blattunterseite befallen, sowie von den Rostpilzen Puccinia milii-effusi DUPIAS (beide Blattseiten, Stängel, Blattstiel) und Triphragmium isopyri MOUG. \& NESTL. (Blattunterseite und Blattstiele). In Gartenkultur leidet die Art unter Schneckenfraß.

\section{Isopyrum thalictroides ${ }^{3}$}

LinNaeus 1753, Sp. Pl. 557. - Syn.: Helleborus thalictroides LAM. 1779, Fl. France 3: 315. Fontanella tertiaria KLUK ex BESSER 1808, Prim. Fl. Galic. 2: 363. - Gontarella tertiaria GILIB. ex STEUD. 1821, Nomencl. Bot., ed. 1, 1: 380. - Isopyrum album DULAC 1867, Fl. Haut. Pyrén. 221. Thalictrella thalictroides (L.) E. NARDI 1993, Webbia 47: 227. - WiesenrautenMuschelblümchen. Franz.: isopyre faux-pigamon; ital.: isopiro comune; slowen.: navadna polžarka; ungar.: galambvirág; slowak.: veterník žltuškovitý; tschech.: zapalice žlut'uchovitá; poln.: zdrojówka rutewkowata; russ.: равноплодник василистниковый [ravnoplodnik vasilistnikovyj]. -

Frühjahrs- bis frühsommergrüne Staude, Geophyt ( $\rightarrow$ Abb. 3), 10-25(-30) cm hoch, ausgedehnte Bestände bildend (,,herdenbildend“). A u s lä u fer - R h i z o m unterirdisch kriechend, dünn ( $\pm 2-3 \mathrm{~mm}$ dick), der Jahreszuwachs 1,5-7cm lang, etwa 4 Jahre erhalten. Bewurzelung sprossbürtig (homorrhiz), Wurzeln zahlreich, fast senkrecht vom Rhizom abstehend, nahe der Basis etwas spindelförmig verdickt; Bewurzelung flach $(<10 \mathrm{~cm})$. S tängel zart, schlank, aufrecht, am Grunde blattlos, meist locker bis dicht zottig behaart, unverzweigt, im oberen Teil außer wenigen Laubblättern den durchblätterten Blütenstand tragend, dort selten auch mit 1-2 Seitenästen, am Grunde von breiten, scheidenartigen, fast weißen Niederblättern umgeben. Nichtblühende Pflanzen stängellos. Grundständige Laubblätter (bei blühenden Pflanzen nur 1-2, wohl zur nächstjährigen Sprossgeneration, bei vegetativen 1-3) kahl bis unterseits locker behaart, bis $12 \mathrm{~cm}$ lang gestielt, doppelt 3-zählig, mit breit verkehrteiförmigen, stumpfen, stumpf gelappten bis handförmig geteilten Abschnitten, diese unterseits blaugrün. Stängelblätter 4-6, den grundständigen Blättern ähnlich, jedoch einfach 3-zählig, kürzer gestielt bis fast ungestielt, nach oben einfacher werdend, die obersten sitzend, 3-zählig, 3-teilig oder nur gelappt, mit zwei 2,5-3 $44 \mathrm{~mm}$ großen, muschelförmigen Pseudostipeln. Blütenstand eine durchblätterte (foliose), 2-10-blütige Traube ohne Endblüte.

3 thalictroides: Thalictrum-ähnlich, wegen der Ähnlichkeit der Blätter mit denen der Wiesenraute 
B lüten 1,2-2,2 cm im Durchmesser, 1-3,5 cm lang gestielt. Äußere Blütenhü1lblätter (= Kelchblätter) kronblattartig, meist (4-)5(-6), 7-11 ×6 mm, verkehrteilänglich, weiß, deutlich geadert, hinfällig. In n e re B 1 ü te n hü 11 blätter (= nektarfertile Kronblätter) 5, etwa 1-2 mm lang, kurz muschelförmig (Abb. 3, 6). Staubblätter zahlreich (etwa 25). F r u c h tb lät te r (1-)2(-5), mit 2reihig angeordneten Samenanlagen und kurzem, gebogenem Griffel. Balgfrüchtchen (oft nur eines reifend) mit dem kurzen, einwärts gebogenem Schnabel (6-) 8-10(-11) $\times 4 \mathrm{~mm}$, länglich eiförmig, seitlich flach zusammengedrückt, abstehend, wenigsamig. S a me n in 2 Reihen, eiförmig, 3-3,2 mm lang, kurz behaart, kastanienbraun ( $\rightarrow$ Abb. 5, KADEREIT \& MELZHEIMER 2021, Tafel 4 Fig. 28, http://www.flora-deutschlands.de/ranunculaceae.html). - Chromosomenzah1: 2n=14. Blütezeit: (II)-III-IV(-V). - Giftig!

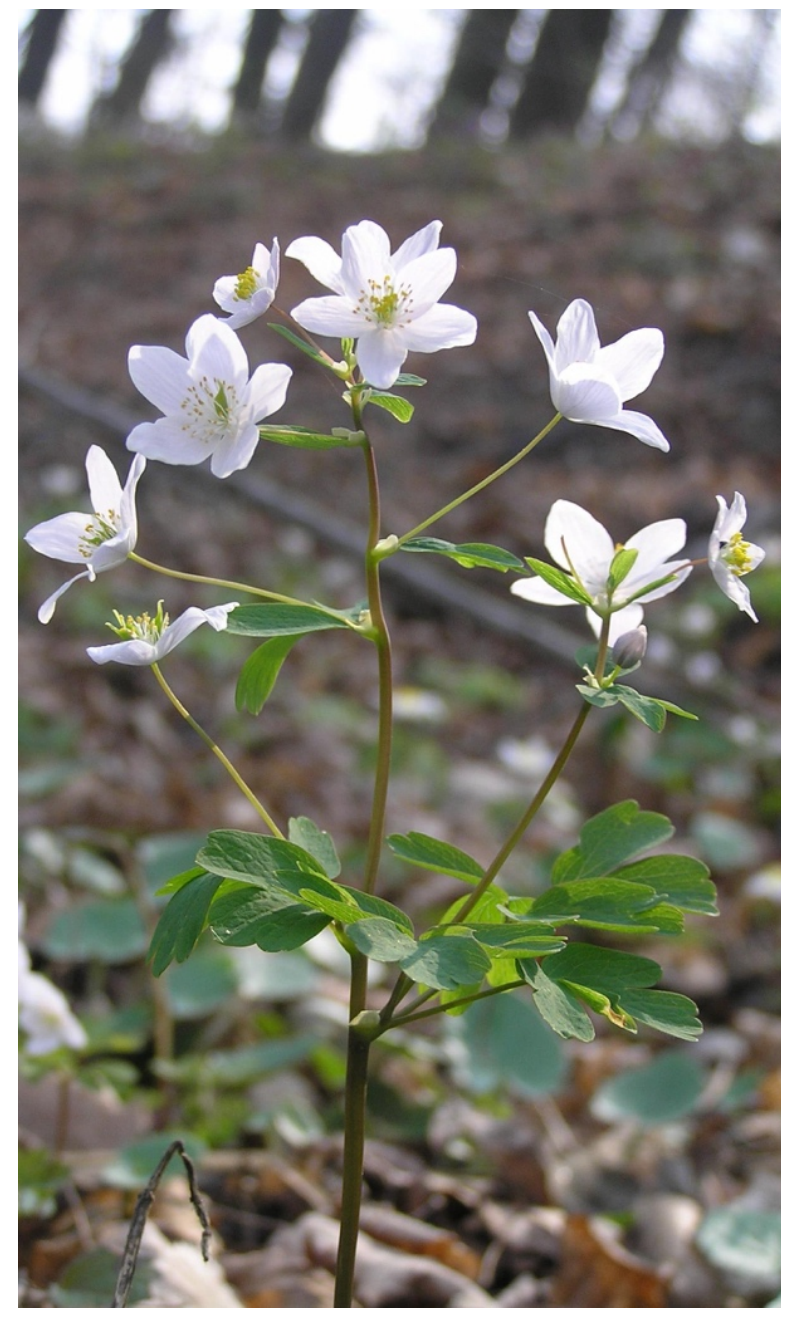

Abb. $2 \mid$ Isopyrum thalictroides L., blühende Pflanze am natürlichen Standort in Nord-Mähren bei Lipnik nad Becvou, 10.04.2009. Blütenstand mit basalem Seitenast (Doppeltraube). Foto: PETR FILIPPOV (GNU-Lizenz für freie Dokumentation)

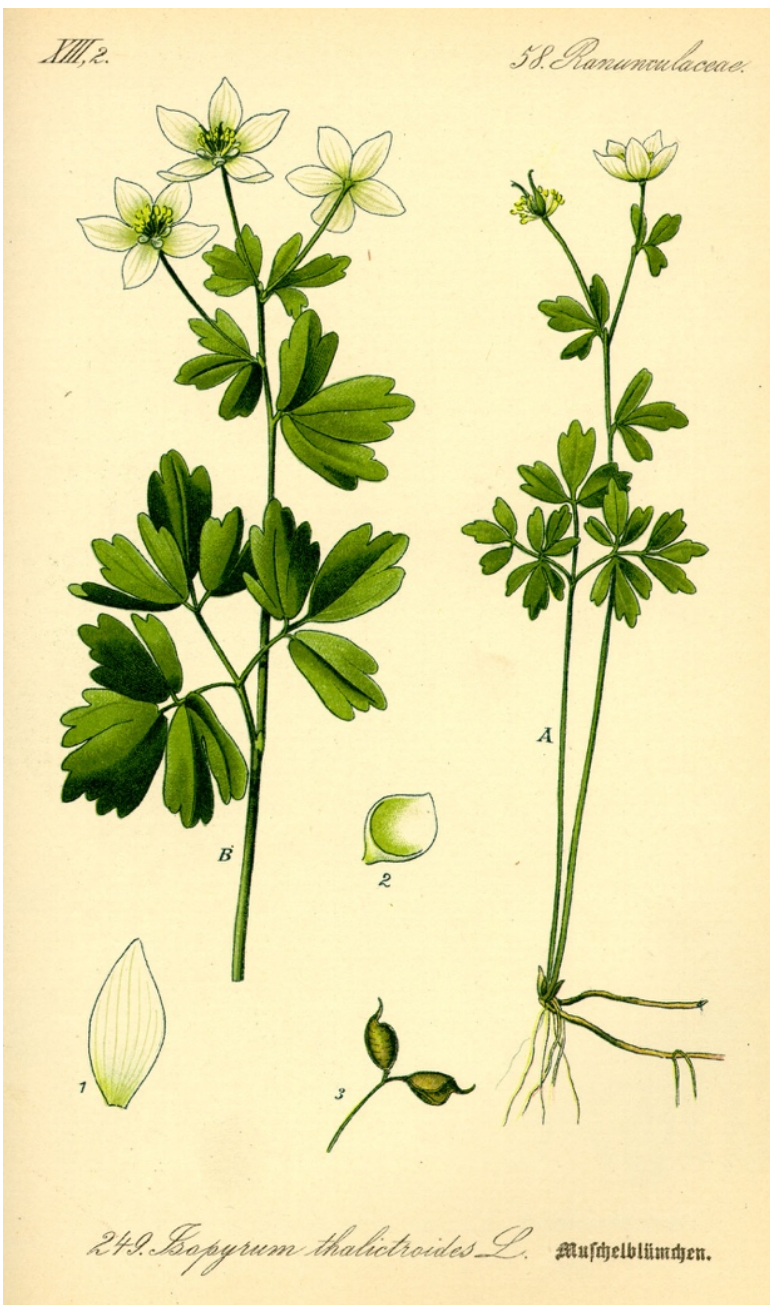

Abb. 3 Isopyrum thalictroides L. Rechts: blühende Pflanze mit unteridischem Ausläufer, links: Bütenstand stärker vergrößert, Nektarblatt, Sammelfrucht mit 2 Bälgen. Aus ThOмÉ, O. W., Flora von Deutschland, Österreich und der Schweiz. GeraUntermhaus 1885. 
Weitere Abbildungen: TROLl \& WEBERLING (1989) $\rightarrow$ Abb. 111. - THOMAS MEYER: Flora-de: Flora von Deutschland (http://www.blumeninschwaben.de/Zweikeimblaettrige/Hahnenfuss/isopyrum.htm). WIKIPEDIA, https://de.wikipedia.org/wiki/Wiesenrauten-Muschelblümchen, Fotos von STEFAN LEFNAER (u. a.).

Standort und Vergesellschaftung. I. thalictroides ist eine hygrophile Laubwaldpflanze, die sehr empfindlich auf Änderungen der Standortsbedingungen reagiert und den Bestand als eines der ersten Elemente verlässt. Zeiger alter Wälder.

Zerstreut, aber bestandsbildend in feuchten, humosen Auenwäldern, in lichten, feuchten Edellaubholzwäldern, an Waldrändern, in bewaldeten Schluchten, an Bergbächen, unter Gebüsch; Verbreitungsschwerpunkt in Eichen-Hainbuchenwäldern (Carpinion-Charakterart, auch in Fagion-Gesellschaften übergreifend). In Böhmen am häufigsten in den feuchtesten Varianten der Assoziation Querco-Carpinetum (Acerenion), die bereits den Übergang zu Auwäldern (Unterverband: Alno-Ulmion) darstellen. In den östlichen Alpen ist Isopyrum besonders in den großen Flusstälern häufig (Mur, Drau, Save, Donau). In den südlichen Kalkalpen Österreichs wird die Pflanze nach VIERHAPPER und HANDELMaZZETTI (in Hegi 1912: Ill. Fl. Mitteleuropa Bd. 3, 1. Aufl. 1912, S. 477) in Wäldern mit Carpinus, Acer pseudoplatanus, Ostrya, Fraxinus ornus, Philadelphus coronarius, Euonymus verrucosus, E. latifolius, Daphne laureola, Staphylea pinnata begleitet von Helleborus niger, H. viridis, Anemone trifolia, Epimedium alpinum, Euphorbia carniolica, Omphalodes verna, Symphytum tuberosum, Pulmonaria vallarsae, Melittis melissophyllum, Lamium orvala, Scrophularia vernalis und Asperula taurina angetroffen, also mit thermo-hygrophilen, z. T. reliktär verbreiteten Laubwald-Elementen. In Nordost-Polen (ehem. Westpreußen) erscheint sie „stellenweise in ungeheurer Menge als entzückende Randeinfassung der Bachufer“. Einen ausgedehnten blühenden Bestand in einem Hang-Waldstandort zusammen mit Anemone ranunculoides bei Mistelbach/Niederösterreich zeigt eine Aufnahme vom 1.4. 2017 von ST. LEFNAER (https://de.wikipedia.org/wiki/Wiesenrauten-Muschelblümchen\#/media/Datei: Anemone ranunculoides + Isopyrum thalictroides sl1.jpg). Von der Ebene bis etwa $1000 \mathrm{~m}$, in den mährisch-schlesischen Beskiden bis $1120 \mathrm{~m}$, in Italien nur bis $500 \mathrm{~m}$.

Zeigerwerte in der Schweiz: Lichtzahl 2 (schattig), Temperaturzahl 4+ (warm-kollin), Feuchtezahl 3+ (feucht), Reaktionszahl 4 (neutral bis basisch), Nährstoffzahl 3 (mäßig nährstoffreich).

Allgemeine Verbreitung. ( $\rightarrow$ Abb. 4): Ostkantabrische Gebirge, Pyrenäen, West- und Zentralfrankreich bis Calvados und Paris, Savoyen, West-Schweiz, Südalpenrand vor allem östlich des Piave, nördlicher Apennin, Apennin von Toskana-Emilia und (?)Latium (von Süd-Korsika irrtümlich angegeben), Sardinien, östliches Mitteleuropa von Oberösterreich über Böhmen, Mähren, die Slowakei, Ungarn, Polen (von Schlesien bis Ostpommern), Russland: Oblast Kaliningrad, bis Südwest-Litauen (Verbreitungslücke im zentralen Mitteleuropa), Ost- und Südosteuropa ostwärts bis Bosnien Montenegro, Bulgarien, Mazedonien, Rumänien, Moldau-Republik, S- und W-Weißrussland (selten, vom Aussterben bedroht) und die südliche und westliche Ukraine (Dnjepr-Gebiet). Verwildert auch im Moskauer botanischen Garten in einem Eichenwald, im Distrikt Kaluga, In N-Bayern (Hof) und in Dänemark. Im Herbarium Wien liegt ein Beleg aus Griechenland: Arkadien: Kynourias $37^{\circ} 16^{\prime} / 22^{\circ} 8^{\prime}$, der nicht in die Floren Eingang gefunden hat. 


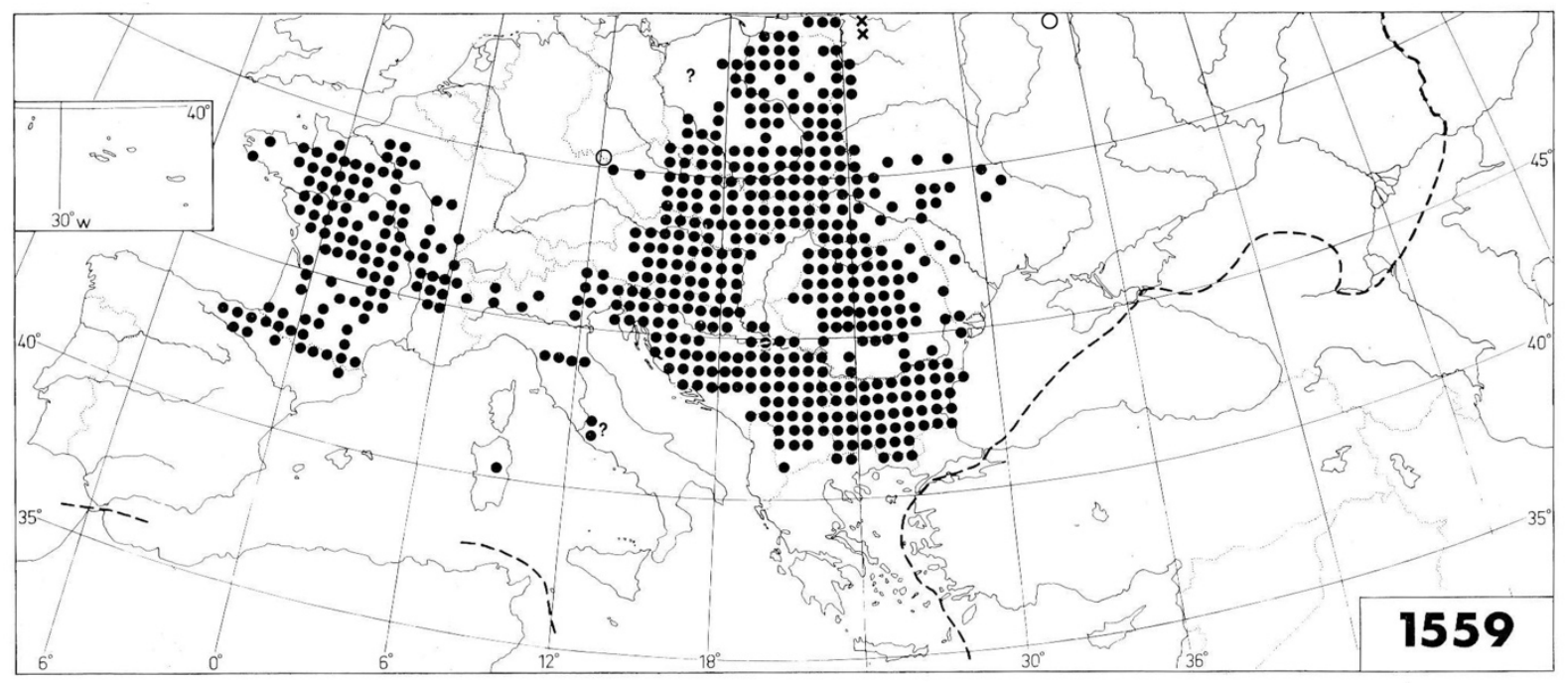

\begin{abstract}
Abb. 4 | Gesamtareal von Isopyrum thalictroides L. Aus: Jalas, J. \& SuOminen, J. 1989: Atlas Florae Europaeae (AFE) 8, S.39. Helsinki, Committee for mapping the Flora of Europe; verändert und ergänzt (Liegende Kreuze: seit 1930 nicht angegeben, wahrscheinlich erloschen. Offene Kreise: Synanthrope Vorkommen in Hof (N-Bayern) und (Status ungeklärt) im Gebiet Kaluga (neu für Russland)). Korsika gelöscht (nach Euro+MEd 2000- beruht die Angabe (LiTARdiÉRE, R., Candollea 15: 27) auf einem Irrtum: http://ww2.bgbm.org/EuroPlusMed/). Neu in Sardinien: https://inaturalist.nz/observations/4112222 First record for Sardegna, 15.06.2014, leg. et det. G. FARRIS, 1660 m a.s.1. (Desulo, Nuoro Prov.; Glechomo-sardoaeAlnetum glutinosae). Nach AFE in der Schweiz erloschen (nur noch synanthrop), nach https://www.infoflora.ch/de/flora/isopyrum-thalictroides.html\#map noch spontan um Genf. Zwei Vorkommen bei Karlovy Vary in Tschechien ergänzt nach: SLAVÍK, B. (1986): Fytogeografické syntézy ČR, Vol. 1; s. auch https://pladias.cz/en/taxon/overview/Isopyrum thalictroides. Fragezeichen am unteren Tiber (Italien, Angaben aus AFE): die Pflanze fehlt nach FARRIS (s. oben) südlich der Toscana. Zwei Vorkommen in der Bretagne (Frankreich) ergänzt nach GBIF: https://www.gbif.org/species/5615962. Vorkommen südlich des Ohrid-Sees (Mazedonien) ergänzt nach: TEOFILOVSKI, A. (2018); Chorological data for some new, doubtfully known and rare plants in the flora of the Republic of Macedonia. Acta Musei Macedonici Scientiarum Naturalium 21/1: 13-22. Weitere Karten aus dem Gebiet: Polen: https://atlas-roslin.pl./gatunki/Isopyrum thalictroides.htm; Friaul: http://ryades.units.it/dryades/plants/foto/VR000845.jpg; Belarus: https://redbook.minpriroda.gov.by.
\end{abstract}

Verbreitungskarten: s. Legende $\mathrm{zu} \rightarrow$ Abb. 4). Die auffällige Verbreitungslücke zwischen dem südatlanischen und ostzentraleuropäischen Teilareal ist weder historisch noch ökogeographisch leicht zu erklären, da sich die Pflanze vom vermutlichen Laubwaldrefugium im perikarpatischen Gebiet etwa $1000 \mathrm{~km}$ nach Norden, aber nach Nordwesten nur 500 km bis Böhmen ausgebreitet hat. Als „Langsamwanderer" könnte sie das potentielle Areal noch nicht ausgefüllt haben, sie verwildert aber auch nur selten aus der Kultur und breitet sich nicht weiter aus.

Zonale Arealdiagnose: sm-temp· $\mathrm{OZ}_{(1-2)-3} \mathrm{EUR}$

Regionale Arealdiagnose: pyr/mo-südatl-zentralsubmed-balc-bohem-polon-westsarm

Verbreitung im Gebiet. In Deutschland spontan fehlend, synanthrop sehr selten, z. B. Bayern (Park Theresienstein in Hof). Vor allem in den östlichen Teilen Zentraleuropas, so in Russland im Süden des Oblast Kaliningrad. z. B. Nesterov [Stallupönen, Ebenrode], Bagrationowsk [Preußisch-Eylau], Käuxertal 
bei Slawskoje [Kreuzberg]. In Polen z. B. im ehem. Ostpreußen im Drengfurt im Kreis Rastenburg, bei Rastenburg [Kętrzyn], im Guttstadt [Dobre Miasto], Glottauer Wald im Kreis Heilsberg [Lidzbark Warmiński], im Kreis Mohrungen [Morąg], Buchwalder Forst im Kreis Allenstein [Olsztyn] und zwischen Schönkau und Wilmsdorf im Kreis Neidenburg [Nidzica]. Im ehem. Westpreußen im Kreis Thorn [Toruń], an der Weichsel, weiter flusabwärts bei Rozanno im Kreis Bromberg [Bydgoszcz], zwischen Bogdanken und Mendritz im Kreis Graudenz [Grudziądz], im Kreis Kulm [Chełmno], Lubochin im Kreis Schwetz [Świecie], Trankwitz bei Christburg im Kreis Stuhm [Sztum], Fürstenau im Kreis Elbing [Elblag]. Ferner bei Koźmin im Kreis Posen [Poznań]. Crone a. d. Brahe und Getau im Kreis Inowrazlaw, zwischen Wroble und Pieranic am Goplo-Bruch, Niedermühl. Häufiger ist die Art im Bereich des Flusses Biebrza, zwischen den Städten Łomza an der Narew und Augustów. Ein weiterer Schwerpunkt liegt im Bereich der südlichen Mittelgebirge, den polnischen Anteilen an den Sudeten, Beskiden und Karpaten. Weitere Nachweise beziehen sich z. B. auf Orte in Niederschlesien, z. B. bei den Berghäusern um Liegnitz [Legnica], vom südlichen Mittelschlesien ostwärts bis Oberschlesien, vor allem in gebirgigen Lagen, z. B. am Königshainer Spitzberg, Silberberg [Srebrna Góra], Glatz [Kłodzko], Teschen [Cieszyn], Bielitz-Biala [Bielsko-Biala] (Karte für Polen: https://atlasroslin.pl./gatunki/Isopyrum thalictroides.htm). In Tschechien (Karte: https://pladias.cz/en/taxon/overview/Isopyrum thalictroides Slavík) in Mittel (Moldau) und Nordostböhmen, Böhmischer Karst, Böhmisches Paradies, Vorland des Riesengebirges, des Adlergebirges und der Sudeten, des Jeschken und Schneebergs, mittleres und östliches Elbe-Gebiet, in Mittelböhmen [Křivoklát] (MLADY \& KOLBEK 1976) vereinzelt bei Landskron [Lanškroun], in Ostböhmen Turnau [Turnov], Jaromierz, Reichenau an der Kněžna [Rychnov nad Kněžna], bei Medo-Oujezd, Brasic bei Colcuv-Jenikov usw. In Nordmähren häufiger z. B. Weidenau [Vidnava], Jägerndorf [Krnov], Troppau [Opava], Stauding [Studénka], in den böhmisch-mährischen Höhen, den mittelmährischen und Weißen Karpaten und dem Beskiden-Vorland. In Österreich collin bis submontan, im Burgenland im Leithagebirge, Rosalien- und ÖdenburgerGebirge; Wien, in Niederösterreich: Wienerwald, Alpenvorland, Donau-Auen, südlichster Teil des Waldviertels, ganzes Weinviertel, Hainburger Berge, Ellender Wald, Lichtenwörther Au, Bucklige Welt. In Oberösterreich von Steyr nach Rosenegg, um Windischgarsten. In der Obersteiermark ziemlich verbreitet, so im Ennstal um Admont, z. B. unter der Kemetenmauer, und von Admont bis ins Gesäuse, im Sunk bei Trieben, im oberen Murtal, bei Judenburg, im Feeberggraben und Reiflinggraben, bei Leoben, Bruck, und im ganzen Mürztale bis auf den Semmering, in Mittelsteiermark bei Piber nächst Voitsberg, im Laßnitztal bei Deutschlandsberg, Eibiswald, in der Grazer Bucht, z. B. mehrfach um Graz und zerstreut im unteren Mur-Gebiet bis In Kärnten im Klagenfurter Becken, Sattnitz gegen Gurnitz, unterhalb St. Martin im Granitztal, an der Lavant bei Wolfsberg. In Vorarlberg ruderal bei ReichenfeldFeldkirch (ob noch?). In den Tiroler Apen fehlt die Art, ebenso in der östlichen und mittleren Schweiz. In Italien am Südalpenrand in den Provinzen Bergamo (ob im Gebiet auch westlicher?), Brescia, Verona, Belluno, Treviso, Pordenone und Udine, häufig besonders östlich des Piave. In Piemont: Biellese nördl. Ivrea (ob noch?). In der Schweiz im Kanton Genf bei Genf und Chancy; im Kanton Waadt eingebürgert bei Nyon und spontan (oder alteingebürgert) bei Les Devens ob Bex; eingebürgert an der Rhone östlich Monthey. In Frankreich im Hegi-Gebiet in Haute Savoie und im Jura. In Slowenien in allen Teilgebieten der Flora verbreitet, z. B. im Drau-, Sann- und Save-Gebiet, BacherGebirge (Pohorje), bei Ptuj [Pettau], Slovenska Bistrica [Windisch-Feistritz], ferner bei Grobelno, Štore, Celje [Cilli], Slovenske Konjice [Gonobitz], Zidani Most [Steinbrück], Kozje [Drachenburg].

Naturschutz und Gefährdung. In Österreich in den Alpen und im Alpenvorland gefährdet. In der Schweiz stark gefährdet (vulnerabel) und vollständig geschützt. In Tschechien, Polen, Italien und Slowenien nicht unter Naturschutz. - Als Zeiger alter Wälder ist die Art durch Intensivierung der Waldnutzung, Rodung von Hecken, Ackerbau bis direkt an den Waldrand und Straßenbau gefährdet. 
Variabilität. Die Art ist im Gebiet hinsichtlich der Behaarung veränderlich. Am Grunde des Stängels und auf den Blattunterseiten ist die Pflanze locker behaart. Allerdings verliert sich die Behaarung bei älteren Pflanzen und vor allem an herbarisierten Pflanzen. Die Behaarung kann sogar weißfilzig am Stängelgrund und locker flaumhaarig auf den Blattunterseiten ausfallen (var. pubescens WIERZB., so im Grazer Bergland und im österreichischen Alpenvorland), MELZER (1984) möchte die Sippe evtl. höher (Unterart?) bewerten.

Cytologie. Als einzige Abweichung von der diploiden Zahl 2n = 14 liegt eine Zählung von $6 n=42$ für eine zentralfranzösische Population vor (K. P. BUTTLER, mündliche Mitteilung März 1998).

Blütenökologie. Die Blüten sind mehr oder weniger homogam bis schwach proterandrisch.

Lebensgeschichte und Jahreszyklus. Die Samen des Muschelblümchens reifen Ende Mai bis Ende Juni und fallen aus den (1-)2(-5) Balgfrüchtchen aus. Ihre Ausbreitungsdistanz ist gering, die Ausbreitungsweise wird als Barochorie bezeichnet (Ausbreitung durch Herabfallen). Die Samen sind rundlich-ellipsoidisch, etwa $3 \mathrm{~mm}$ lang und 1,5 mm dick ( $\rightarrow$ Abb. 5), das Tausendkorngewicht beträgt 5,3 g. Wahrscheinlich sind auch Ameisen an der Ausbreitung beteiligt (Myrmekochorie).

Das Foto eines Samenkorns $(\rightarrow$ Abb. 5) zeigt nämlich ein Anhängsel an der Mikropyle, und da die Samen hypogäisch keimen, wohl auch Dunkelkeimer sind, müssen sie unter die Erdoberfläche gelangen. Sie keimen aber nicht im Jahr der Reife, sondern erst im nächsten Frühjahr. Als Kaltkeimer brauchen die Samen für die Entwicklung des Embryos zunächst eine Periode von 2-4 Wochen, in der der Boden feucht und warm ist (Lufttemperatur $\left.18-22^{\circ}\right)$, danach aber eine wenigstens 4-6-wöchige Kälteperiode $\left(-5^{\circ}\right.$ bis $\left.+5^{\circ} \mathrm{C}\right)$. Das entspricht dem subozeanisch-subkontinentalen Areal der Art, in dem sommerlich feuchtwarme Bedingungen und langdauernde Winterkälte abwechseln.

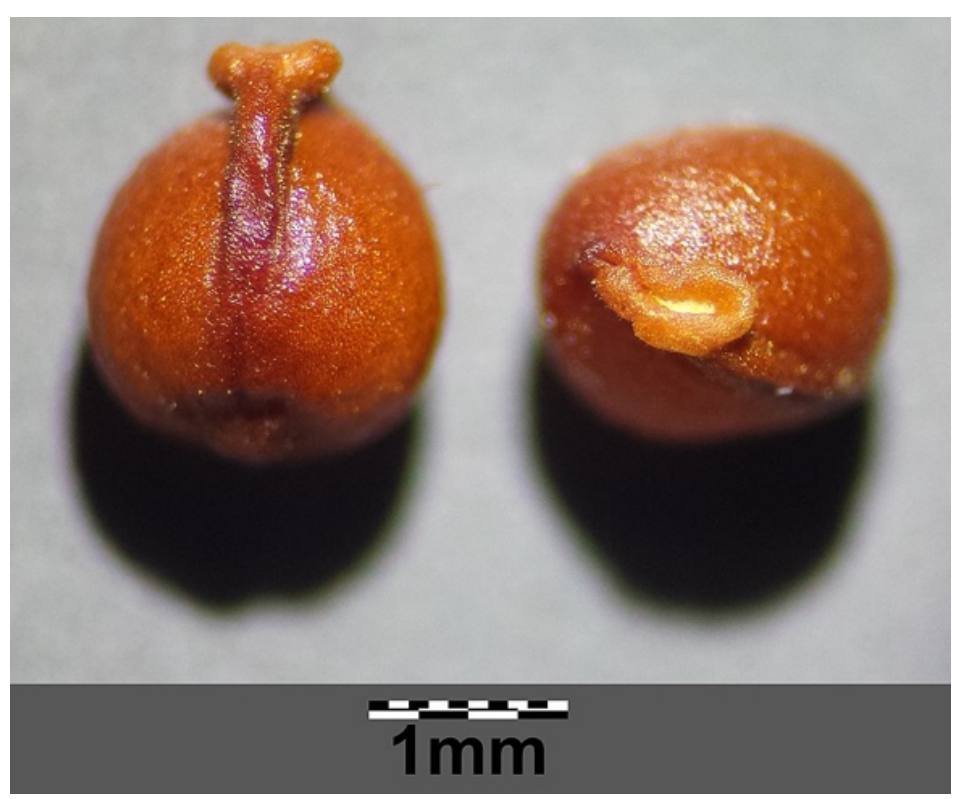

Abb. 5: Samenkorn von Isopyrum thalictroides L. gesammelt am 25.05.2015 bei Niederhollabrunn/ Niederösterreich. Aufn. 15.12.2015. Foto: STEFAN LEFNAER. Aus Wikipedia 2021.

Erst im Frühjahr wird die Samenschale gesprengt. Die beiden halbkugeligen Keimblätter verbleiben im Boden (hypogäische Keimung), sie biegen sich zurück, geben den Vegetationspunkt frei und ernähren den Keimling bis zur Bildung eines ersten kleinen Primärblattes und eines Kranzes von etwa 6 basal verdickten sprossbürtigen Wurzeln aus dem Keimblattknoten $(\rightarrow$ Abb. 6). Die Keimwurzel und das Hypocotyl gehen bald zugrunde. Auch später wird an jedem Niederblatt- und Laubblattknoten des Erdsprosses ein Kranz von sprossbürtigen Wurzeln ausgebildet. Die Bewurzelung ist also (wie bei den meisten Monocotyledonen) von Anfang an sprossbürtig, mindestens bis zum 4. Jahr knotenbürtig, nach KÄSTNER \& FISCHER (2006) ( $\rightarrow$ s. Abb. 7) später auch internodienbürtig. 


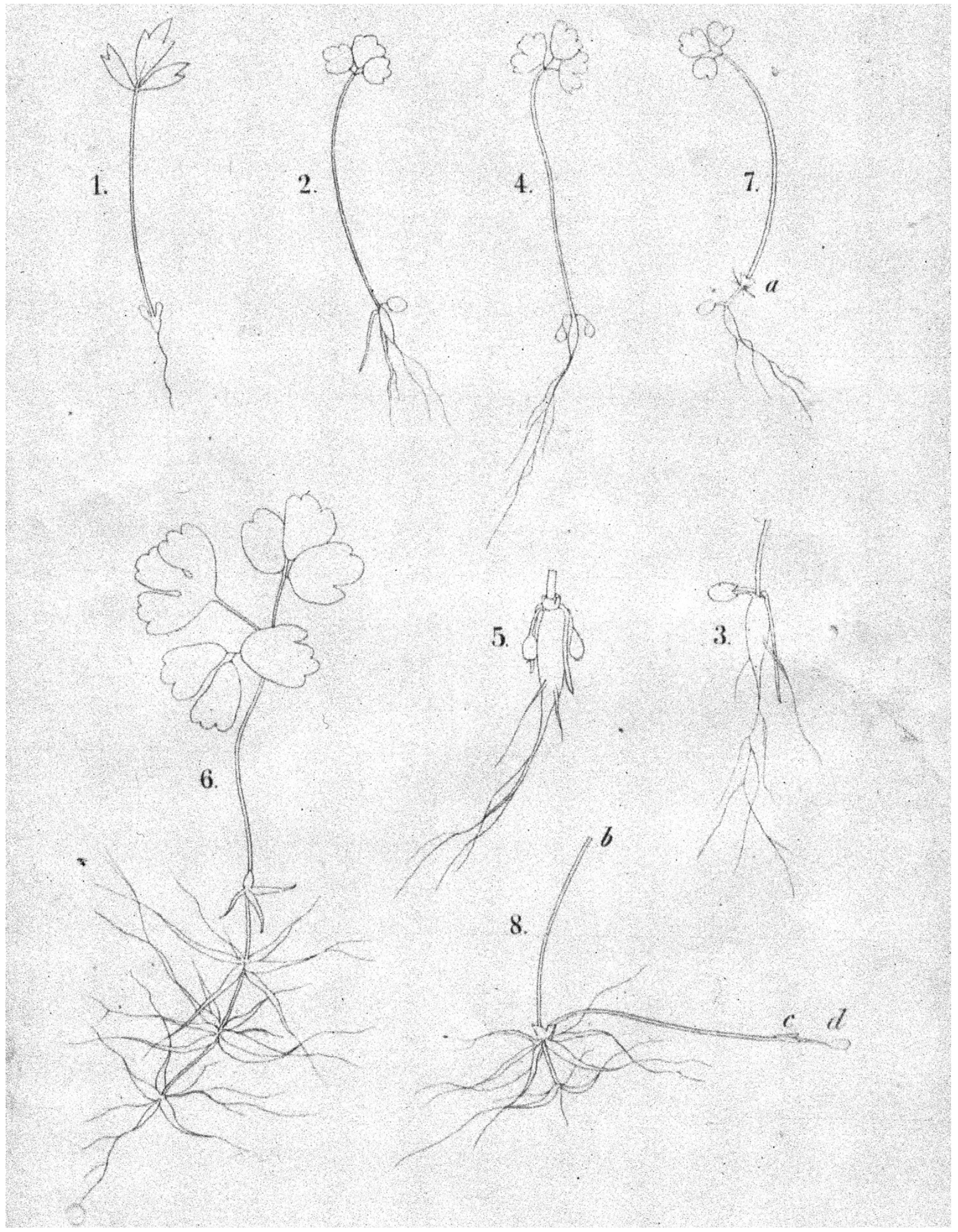

Abb. $6 \mid$ Keimling und Jungpflanze von Isopyrum thalictroides L. 1: Keimpflanze der Anemone nemorosa (zum Vergleich) Nat. Gr. - 2. Keimpflanze des Isopyrum mit den in der Samenschale eingeschlossenen Keimblättern. Nat. Gr. - 3. Der untere Teil einer anderen, etwas vergrößert. - 4. Keimpflanze, nach Ablösung der Samenschale. Nat. Gr. - 5. Der untere Teil, etwas vergrößert. - 6. Eine wahrscheinlich vierjährige Pflanze mit etagenweise stehenden Wurzelbüscheln. - 7. Keimpflanze mit gestreckter Hauptachse und dem Beginnen eines zweiten Wurzelbüschels (bei a). Nat. Gr. - 8. Hervorgebrochener vegetativer Spross. Bei b abgeschnittenes Blatt, d die Endknospe. Nat. Gr. Aus: WinkLER, A. (1884): Die Keimpflanze des Isopyrum thalictroides L. - Flora 67: 195-198. 


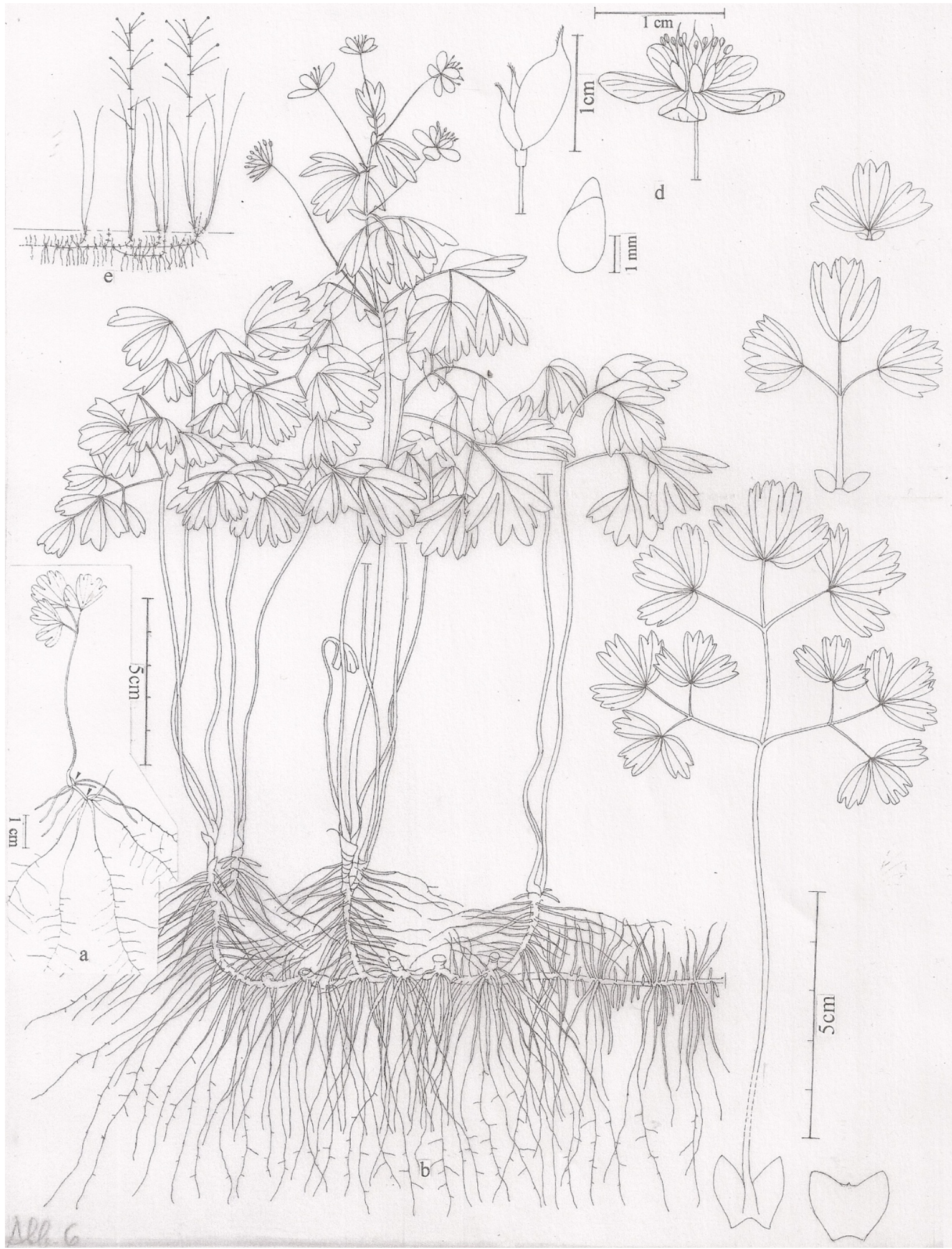

Abb. 7 Isopyrum thalictroides, a Jungpflanze aus Topfkultur; Aussaat Februar 2002, gezeichnet am 15.04.2003 (im 2. Jahr). Bewurzelung nur sprossbürtig aus den Sprossknoten, Wurzeln an der Basis verdickt. - b: Adulte Pflanze vom natürlichen Standort (Petronell. östl Niederösterreich) Rhizomabschnitte dicht sprossbürtig bewurzelt, Wurzeln und Rhizomabschnitte mindestens 4 Jahre lebend. Rechts: Blattfolge, Grundblatt, grundständiges Blatt und stängelständiges Laubblatt, an der Basis Pseudostipeln. - d: Blüte mit den muschelförmigen Nektarblättern, Sammelbalgfrucht und Samen (?, $\rightarrow$ vgl. Abb. 5!). e: Wuchsformschema, Zeichnung: A. KÄSTNER. Aus KäSTNER \& FiSCHER (2006). 
Die Bewurzelungstiefe ist gering (wenige $\mathrm{cm}$, Mullbodenwurzler), die Ausläufer liegen 2-3 cm tief, ihr Jahres-Zuwachs ist 3-10 cm lang und im Juli fertig ausgebildet. Das Jugendstadium, in dem jährlich am Ausläufer-Ende außer Niederblättern nur 1(-2-3?) grundständige Laubblätter ausgebildet werden, dauert etwa 4 Jahre. Der Stängel der blühreifen Pflanze ist unten blattlos, 1(-2?) grundständige Blätter gehören schon zum Innovationstrieb. Der Austrieb erfogt im März; nach der Blütezeit im (Februar-)MärzApril(-Mai) reifen die Früchtchen Ende Mai bis Juni, und Anfang Juli stirbt die Pflanze oberirdisch ab. Die Samenproduktion ist ziemlich gering (etwa (5-) 10-25 Samen pro Pflanze (Ramet) im Jahr) da in den meist 2 Balgfrüchtchen nur 2-6 Samen reifen. Die Lebensdauer der Samen ist nicht bekannt. Die Lebensdauer der Pflanze ist an einem geeigneten Standort durch das Wachstum ihrer AusläuferRhizome unbegrenzt.

Die eigenartige Beschränkung auf jährlich ein einziges grundständiges Laubblatt während des juvenilen Stadiums ist bei mehreren Ranunculaceen zu beobachten, z. B. bei Eranthis, Ranunculus thora und $R$. hybridus, bei mehreren Anemone-Arten (A. nemorosa, A. ranunculoides u.a.), wohl auch bei Actaea spicata, aber auch bei Liliaceen (Tulipa), dagegen nicht bei den stärker abgeleiteten Familien im System. Am Ende des Jugendstadiums wird ein beblätterter Blütenstängel angelegt, grundständige Blätter stehen dann nur am Innovationsspross. Die Dauer des einblättrigen Jugendstadiums hängt stark von der Gunst des Standorts ab, sie beträgt bei Eranthis hyemalis meist 4-6 Jahre, auch bei I. thalictroides werden 4 Jahre angegeben, aber viele Pflanzen verharren viel länger im blütenlosen einblättrigen Stadium. Bei Tulipa sylvestris wurden in einem großen Bestand im Botanischen Garten Halle viele Pflanzen beobachtet, die über 5 Jahrzehnte im vegetativen Einblattstadium blieben.

Verwendung als Zierpflanze. Das Muschelblümchen wird in England, Frankreich und Deutschland als winterharter Frühjahrsblüher von Staudengärtnereien zur Kultur in Torfbeeten, Gehölzgärten oder Steingärten angeboten (Jelitto: 50 Samen 3,70€, Vermehrung aber leicht durch Teilung.). Auf humusreichem, kalkhaltigem, feuchtem, aber wasserdurchlässigem Boden bildet es durch Ausläufer bodendeckende Bestände. Es ist aber bisher ziemlich selten in Kultur.

Volksnamen (MARZELL 1972). Der Name D old o cke, Tolld ocke scheint schlesisch zu sein. Er tritt gegen Ende des 18. Jahrhunderts im botanischen Schrifttum auf, wird jetzt noch als Tôltucke (Wölfelsgrund/Glatz) angegeben und bezieht sich wohl auf die Giftigkeit. Ähnlich heißt der Germer (Veratrum album) Doltocken, Oldocke, Holtucken. Merkwürdig ist auch der schlesische Name Liebenötze. Die Bezeichnung Muschelblümchen ist künstlich gebildet. Der Name bezieht sich auf die Form der Nektarblätter; Usterblamichi (Siebenbürgen) auf die frühe Blütezeit.

Dank: Herrn Dr. C. Berg (Graz) danke ich für die Zusendung von frischem Lebend-Material des Muschelblümchens. Herrn Prof. Dr. J. W. KADEREIT danke ich für die kritische Durchsicht des Manuskriptes, Herrn Dr. E. WELK für Hinweise auf neue Kartierungen, Frau D. FRANKE für die Gestaltung des PDFs. 